\title{
Formation and growth of nanoindentation-induced high pressure phases in crystalline and amorphous silicon
}

\author{
S. Ruffell, ${ }^{\text {a) }}$ J. E. Bradby, and J. S. Williams \\ Department of Electronic Materials Engineering, Research School of Physical Sciences and Engineering, \\ Australian National University, Canberra, Australian Capital Territory, 0200, Australia \\ P. Munroe \\ Electron Microscope Unit, University of New South Wales, Sydney, New South Wales, 2052, Australia
}

(Received 18 June 2007; accepted 26 July 2007; published online 26 September 2007)

\begin{abstract}
Nanoindentation-induced formation of high pressure crystalline phases (Si-III and Si-XII) during unloading has been studied by Raman micro-spectroscopy, cross-sectional transmission electron microscopy (XTEM), and postindentation electrical measurements. For indentation in crystalline silicon $(c-\mathrm{Si})$, rapid unloading $(\sim 1000 \mathrm{mN} / \mathrm{s})$ results in the formation of amorphous silicon $(a$-Si) only; a result we have exploited to quench the formation of high pressure phases at various stages during unloading to study their formation and evolution. This reveals that seed volumes of Si-III and Si-XII form during the early stages of unloading with substantial volumes only forming after the pop-out event that occurs at about $50 \%$ of the maximum load. In contrast, high pressure phases form much more readily in an $a$-Si matrix, with substantial volumes forming without an observable pop-out event with rapid unloading. Postindentation electrical measurements have been used to further investigate the end phases and to identify differences between indentations which otherwise appear to be identical from the XTEM and Raman analyses. () 2007 American Institute of Physics.
\end{abstract}

[DOI: $10.1063 / 1.2781394]$

\section{INTRODUCTION}

Nanoindentation-induced phase transformations in silicon have attracted significant interest over recent years. High pressure diamond anvil and indentation experiments have revealed much about the behavior of silicon under these loading conditions. ${ }^{1-3}$ On loading, a transformation from crystalline $\mathrm{Si}-\mathrm{I}(c-\mathrm{Si})$ to a conducting metallic phase ( $\mathrm{Si}-\mathrm{II})$ occurs at a critical pressure of $\sim 12 \mathrm{GPa}$. For diamond anvil experiments, in the case of $c$-Si samples, this phase has been observed to transform to $\mathrm{Si}$-XII and then $\mathrm{Si}$-III during pressure release as the metallic phase is unstable below pressures of $\sim 8 \mathrm{GPa}^{2,3}$ Indentation experiments have revealed further possible phase transformations on unloading. For fast unloading, the metallic phase transforms to amorphous silicon ( $a-\mathrm{Si}$ ), but on slow unloading it transforms to a mixture of high pressure polycrystalline phases (Si-III and Si-XII). ${ }^{4-6}$ These pressure-induced transformations are of great interest and workers have investigated the transformation pathways, ${ }^{7-14}$ but the exact mechanisms behind the phenomena are still not well understood.

Recently, it has been shown that ion-implanted $a$-Si undergoes similar phase transformations and it has been suggested that the formation and evolution of the high pressure crystalline phases on unloading differ somewhat from $c-\mathrm{Si}^{15}$ Haberl et al. ${ }^{16}$ and Ruffell et al. ${ }^{15}$ have studied these phase transformations during indentation in relaxed $a-\mathrm{Si}$ (formed by ion-implantation and subsequent annealing at $450{ }^{\circ} \mathrm{C}$ ) via a combination of Raman micro-spectroscopy (referred to as Raman hereafter) and cross-sectional transmission electron

\footnotetext{
${ }^{a)}$ Electronic mail: simon.ruffell@anu.edu.au
}

microscopy (XTEM), as well as in situ electrical characterization previously used for studying nanoindentation in $c$-Si. ${ }^{9}$ They found that both materials undergo similar phase transformations during loading and subsequent unloading. On unloading, however, the high pressure crystalline phases (Si-III/ XII) form much more readily during indentation in an $a-\mathrm{Si}$ matrix, e.g., volumes of high pressure phases were formed in $a$-Si with unloading rates at over three orders of magnitude greater than the unload rates required to form these phases in $c$-Si. ${ }^{15}$

In situ monitoring of the high pressure phase formation and evolution is nontrivial and ex situ measurements provide limited information on any phase transformations that occur during unloading. In situ Raman spectroscopy has been demonstrated during indentation of $\mathrm{Si}$, but measurements could only be made on the extruded material and not directly under the indenter tip. ${ }^{17}$ In situ transmission electron microscopy (TEM) has also been demonstrated, ${ }^{18-20}$ but the technique requires an electron transparent sample. In this case it is questionable whether such a thin sample has the same mechanical response as that of bulk silicon. In our case, we have used in situ electrical measurements and the differing electrical properties of the phases to track the phase transformations. ${ }^{21,22}$ The technique is promising in that the electrical properties of the phases under the indenter can be probed directly, but such properties still need to be correlated with both indentation loading data and microstructure.

Previous studies have shown that very rapid unloading $(\sim 1000 \mathrm{mN} / \mathrm{s})$ during indentation of $c$-Si results in preferential formation of $a-\mathrm{Si}$ in the phase transformed zone as measured by both Raman and XTEM. ${ }^{4-6,15,23,24}$ In this study, we take advantage of this as a means of quenching the for- 
mation of high pressure phases at different points on the unloading curve. Postindentation Raman micro-spectroscopy and XTEM can then be used to investigate the formation of high pressure phases (if any) that form prior to the rapid unloading. It should be noted, however, that both techniques have a detection sensitivity threshold below which volumes of high pressure phases may be undetected. Further, high pressure phases form much more readily in $a$-Si so it is not clear that the quenching mechanism works well when indenting silicon in this form, ${ }^{15}$ thus making it more complicated to track the formation of these phases during unloading in this case.

The current study explores differences in the phase transformation behavior between $a-\mathrm{Si}$ and $c$-Si and attempts to investigate the evolution of the high pressure phases during unloading. In addition to XTEM and Raman measurements, in situ electrical measurements have been used to determine differences, if any, between indents (created under different unloading conditions) that are indistinguishable by the XTEM and Raman measurements performed here. Such measurements have been previously shown to be extremely sensitive to the state of the phase transformed silicon. ${ }^{21,22,25}$

\section{EXPERIMENT}

Amorphous and crystalline samples were prepared from Czochralski grown $\mathrm{Si}(100)$ wafers $p$-doped with boron to a resistivity of $8-12 \Omega-\mathrm{cm}$. Approximately $10 \times 10 \mathrm{~mm}^{2}$ samples were cleaved from the wafer and were either indented without further processing or implanted at liquid nitrogen temperature with $\mathrm{Si}$ ions at energies of $50 \mathrm{keV}, 500$ $\mathrm{keV}, 1 \mathrm{MeV}, 1.5 \mathrm{MeV}$, and $2 \mathrm{MeV}$, each to a fluence of 1 $\times 10^{15} \mathrm{~cm}^{-2}$ to produce a surface amorphous layer of thickness $2 \mu \mathrm{m}$. This $a$-Si layer was relaxed by furnace annealing in a flowing argon ambient at $450{ }^{\circ} \mathrm{C}$ for $30 \mathrm{~min}$ prior to indentation.

Indentation was performed at room temperature in air using an Ultra-Micro Indentation System-2000 (UMIS) fitted with a $\sim 4.3 \mu \mathrm{m}$ radius spherical indenter tip. Loading to 80 $\mathrm{mN}$ (resulting in a maximum penetration depth of $\sim 450 \mathrm{~nm}$ ) was performed in 40 increments of $2 \mathrm{mN}$ for all samples. Unloading was allowed to proceed with the same number of increments resulting in an average unload rate of $\sim 0.9 \mathrm{mN} / \mathrm{s}$ until a specified load was reached, at which point rapid unloading to $0 \mathrm{mN}$ at $\sim 1000 \mathrm{mN} / \mathrm{s}$ was performed to quench in the formation of high pressure phases (if any). Under the conditions here, complete unloading at a rate of $0.9 \mathrm{mN} / \mathrm{s}$ ensures that the phase transformed zone will be composed entirely of a mixture of $\mathrm{Si}$-III and $\mathrm{Si}$-XII phases. ${ }^{5,6,15,16,24}$ The rapid unloading positions were chosen to be equal to $100 \%, 90 \%, 70 \%, 50 \%, 20 \%$, and $0 \%$ of the maximum load. From this point on in the article, whenever unloading is referred to as "fast/rapid" the rate is $\sim 1000 \mathrm{mN} / \mathrm{s}$ and "slow" unloading is $\sim 0.9 \mathrm{mN} / \mathrm{s}$. Arrays of each of these indentations were made in both $c$-Si and relaxed $a$-Si substrates.

The phase transformed zones of each of the indents were characterized via Raman micro-spectroscopy using a Renishaw 2000 instrument with a $632.8 \mathrm{~nm}$ wavelength laser

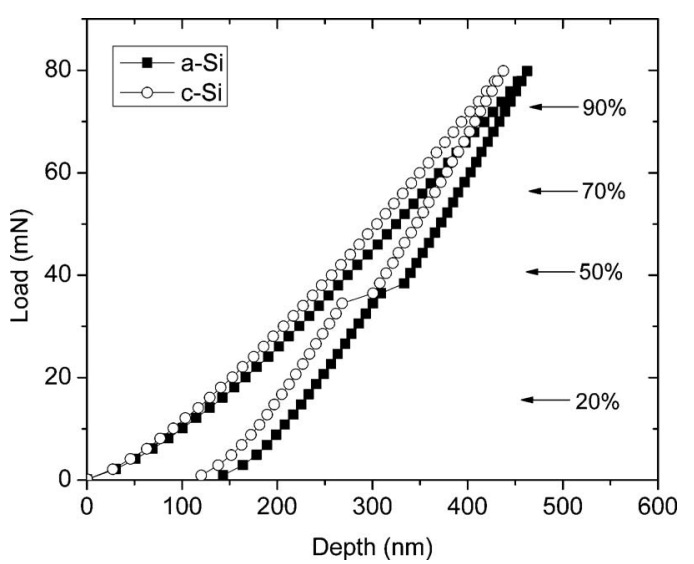

FIG. 1. Load/unload curves from complete indentations in both $c$-Si and relaxed $a$-Si. Loading and unloading were both performed in 40 increments (average rate of $\sim 0.9 \mathrm{mN} / \mathrm{s}$ ). There are slight differences in the mechanical response of the two starting materials but the pop-out event occurs for both at just below $50 \%$ of the maximum load. The positions where rapid unloading was performed are marked by arrows.

focused to a $\sim 1 \mu \mathrm{m}$ spot size. Cross-sectional TEM samples were prepared by a focused ion beam milling technique described elsewhere ${ }^{26,27}$ and imaged using a Philips CM 300 transmission electron microscope. This allowed specific indentations (already measured by Raman) to be imaged by XTEM and correlated with the Raman spectra and the load/unload curves.

Postindentation electrical measurements were made using a conducting diamond Berkovich indenter tip, fitted to a Hysitron Triboindenter, as a point probe, thus allowing precise positioning at points not only in different indents but at specific locations within each residual indent. Using this technique, $I-V$ characteristics were extracted and used to investigate the local structure within the residual indents. This measurement is described in detail elsewhere. ${ }^{21,22}$ In addition, such electrically conducting point probes have previously been used in scanning probe microscopy ${ }^{28-30}$ for dopant profiling, particularly related to shallow $p / n$ junctions. Unfortunately, the through-sample measurement requires a noninsulating sample so measurements in $a$-Si (insulating) samples could not be made.

\section{RESULTS}

Figure 1 shows representative, complete load/unload curves for the indentations performed in both $c$-Si and relaxed $a$-Si. A small difference in the maximum penetration depth is observed reflecting the difference in hardness between $c$-Si and relaxed $a-\mathrm{Si}$ (relaxed $a$-Si is approximately $5 \%$ softer than $c$-Si). ${ }^{31}$ Pop-outs during unloading are observed during indentation in both forms of silicon. The slopes of the unloading curves are similar until later stages of unloading (after pop-out), indicating a greater difference in mechanical response (and phase transformation behavior) at these stages of the indentation cycle. Pop-outs occurred in approximately the same position for both materials on unloading (for all indentations pop-outs occurred after unload- 


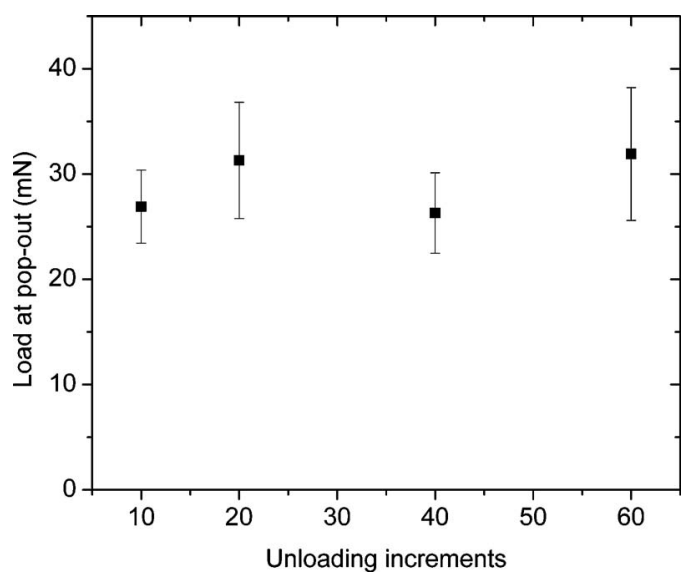

FIG. 2. Load at which pop-out occurred, during unloading from a maximum load of $80 \mathrm{mN}$, versus unload rate (number of increments). For each unload rate, ten indents were performed. For all unloading rates the pop-out occurs at approximately $32 \mathrm{mN}$. For unloading at rates which varied by over an order of magnitude (in a regime where pop-outs occur), the position of pop-out is independent of unload rate.

ing past $50 \%$ of maximum load). The percentages of maximum load at which the rapid unloading was performed are marked.

Pop-outs were found to occur at approximately the same point on the unloading curves independent of the unload rate (at least for rates where a pop-out occurs). Figure 2 shows the position of the pop-out event as a function of unload rate. For unloading from a maximum load of $80 \mathrm{mN}$, using a range of rates which varied by over an order of magnitude (10 to 60 increments), the pop-out always occurred at $32 \pm 7 \mathrm{mN}$.

Raman spectra were collected from indents in both $a-\mathrm{Si}$ and $c$-Si for all unloading conditions. The probability for obtaining high pressure phases for particular unloading conditions was measured by sampling $\sim 25$ indents, made under identical conditions, and determining the presence of such phases by identification of signature peaks associated with $\mathrm{Si}$-III/XII phases in the Raman spectra. Figure 3 shows this probability as a function of the percentage of maximum load where rapid unloading takes place. For complete unloading at the slow rate, all indents measured contained high pressure

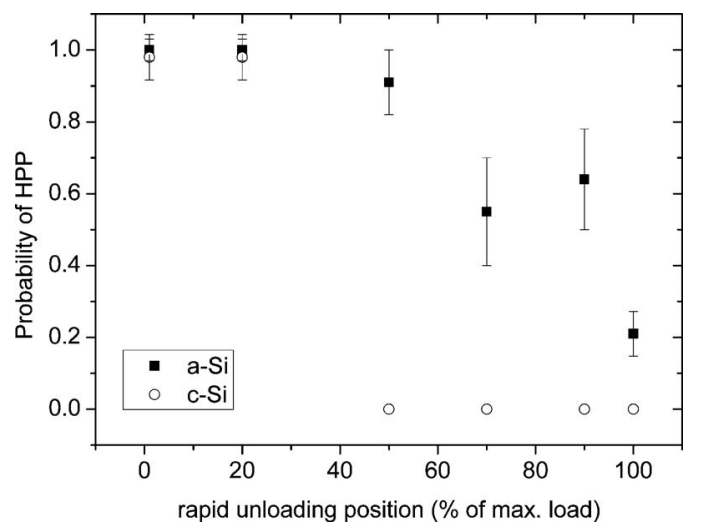

FIG. 3. Summary of Raman data taken on the indents. The probability of high pressure phases (HPP) being detected by Raman is plotted as a function of position of rapid unloading. Error bars are calculated from the number of indents sampled by Raman. phases in both $c$-Si and $a$-Si. Unloading rapidly from the maximum load resulted in no high pressure phases ever being detected in the indents in $c$-Si, but there is an $\sim 20 \%$ chance of them being detected (by Raman) for such indents in $a-\mathrm{Si}$, in agreement with a previous study. ${ }^{15}$ Indeed, for $c$-Si no high pressure phases were detected for indents where rapid unloading took place before a pop-out event. For $a$-Si, the probability of obtaining high pressure phases increases from 0.2 for rapid unloading from maximum load to nearly 1 for unloading rapidly from $50 \%$ of the maximum load.

In addition to detection of high pressure phases, Raman measurements provide the approximate ratio of $\mathrm{Si}$-III to $\mathrm{Si}$ XII through the ratio of the intensity of the Raman peaks at 430 and $350 \mathrm{~cm}^{-1}$. 83 For all samples containing high pressure phases no significant differences in the ratio of these phases were measured in both $a-\mathrm{Si}$ and $c$-Si.

Figure 4 shows XTEM images of indents in $c$-Si where rapid unloading occurred from $100 \%, 90 \%, 70 \%, 20 \%$, and $0 \%$ of the maximum load. The XTEM image from the indent where complete unloading at the slow rate $(0 \%)$ [Fig. 4(e)] was performed is indistinguishable from that where rapid unloading occurred from $20 \%$ of the maximum load [Fig. 4(d)], but shows high volume fractions of the high pressure phases in the phase transformed zone. When rapid unloading is performed from $\geq 50 \%$ of maximum load, the indents are mostly composed of $a$-Si with small volumes of high pressure phases observable by TEM. Selected area diffraction patterns (SADP) verify that these regions are composed of $\mathrm{Si}$-III/XII. Figure 5 shows XTEM images that highlight selected $\mathrm{Si}$-III/XII grains taken from indents in which small volumes of high pressure phases are detected (rapid unloading from $\geq 50 \%$ of maximum load). For all indents created under these conditions and sampled by Raman, no high pressure phases were detected (see Fig. 3), indicating the limited sensitivity of Raman in such cases. When rapid unloading occurs after the pop-out ( $<50 \%$ of max. load), substantial volumes of high pressure phases are detected both by XTEM and Raman. In addition, the residual indentation depth (at zero load) is greater for these indents than those with substantial $a$-Si volumes, and this is clearly noticeable in the XTEM images. The density of the high pressure crystalline phases is higher than that of $a-\mathrm{Si}$ (Ref. 9), so it is expected that the residual depth of the indentation increases with increasing volume of the high pressure phases. No variation in the residual depth is observable in the XTEM for the indents in which rapid unloading occurred before the pop-out event. This is further evidence that there are only small volumes of the high pressure phases within a mostly $a$-Si phase transformed zone in these cases.

Figure 6 shows similar XTEM data for indentations in relaxed $a$-Si. For all the indents shown, high pressure phases were detected by Raman. A substantial volume of these phases is observed (and verified by SADP) even for those cases rapidly unloaded from the maximum load. For all indents in $a$-Si the high pressure phases are located below the surface and extend approximately to the periphery of where the Si-II phase forms at maximum load. After unloading rap- 


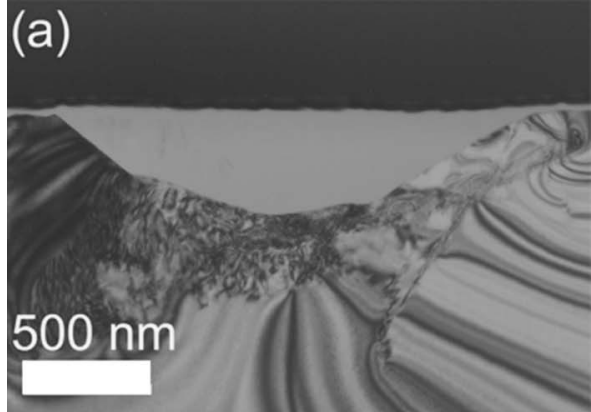

(b)
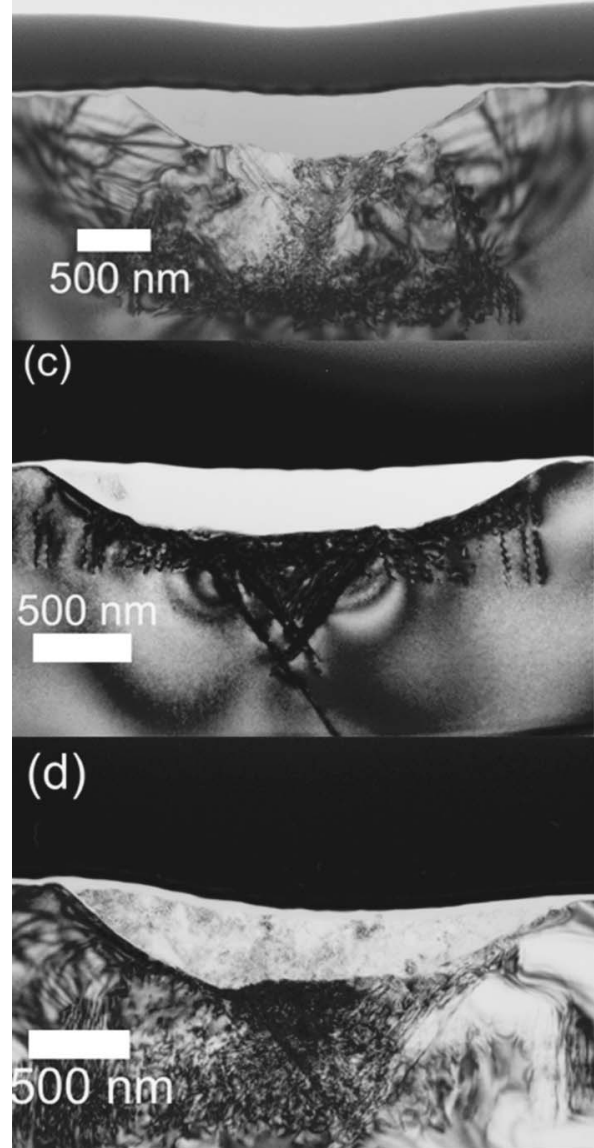

(e)

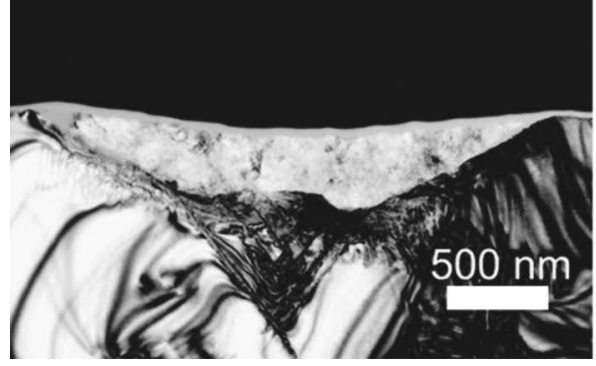

FIG. 4. Bright field XTEM images from indents in $c$-Si. The indents were created by unloading from (a) $100 \%$, (b) $90 \%$, (c) $70 \%$, (d) $20 \%$, and (e) $0 \%$ (complete unloading) of the maximum load. Small volumes of high pressure phases are observable in the indents created by unloading from $\geq 50 \%$ of the maximum load. After the pop-out event, the indents are indistinguishable and are composed mostly of high pressure phases (little evidence of $a$-Si is observed)

idly from $<70 \%$ of the maximum load, the phase transformed zones are composed almost completely of high pressure phases.

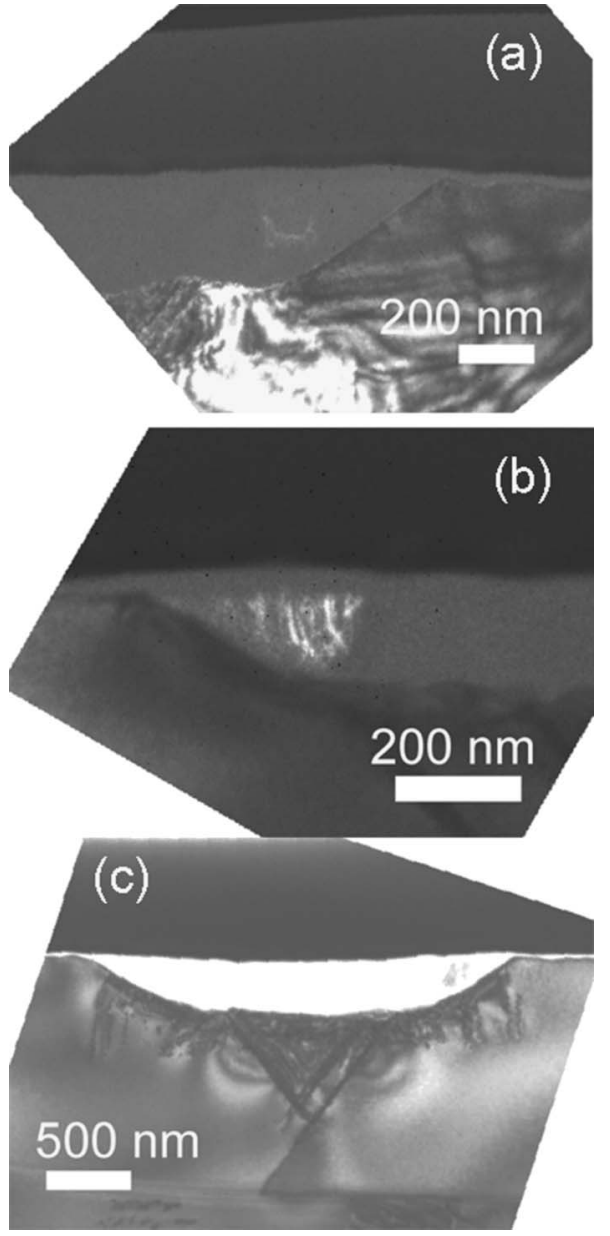

FIG. 5. Dark field XTEM images taken from indents in $c$-Si where rapid unloading occurred from (a) $90 \%$, (b) $70 \%$, and (c) $50 \%$ of maximum load. These images highlight the small volumes of high pressure phases that form within an $a$-Si matrix during early stages of unloading.

Figure 7 shows $I-V$ characteristics extracted from indents in $c$-Si where either slow unloading was performed [Fig. $7($ a)] or rapid unloading only from $20 \%$ of maximum load [Fig. 7(b)]. Multiple $I-V$ measurements were made at different locations within an indent and multiple indents were probed. For both sets of indents, there is a variability in electrical characteristics (under forward bias) measured both within each residual indent (seen in repeat measurements at different locations within same indent) and for different indents. Presumably, this variability in the conductivity measured under forward bias results from variations in the high pressure phase microstructure (and possibly volume ratios of Si-III/Si-XII phases) both within and between indents. Therefore, it is impossible to determine overall differences between indents unloaded completely or unloaded rapidly from $20 \%$ of the maximum load. This is also the case for Raman and XTEM results, indicating that microstructural variations that arise from the statistical nature of nucleation and growth of high pressure phases during unloading mask any differences between slow unloading and fast unloading from the latter position of the load/unload curve.

Figure 8 shows $I-V$ characteristics extracted from indents in $c$-Si where rapid unloading occurred before the popout. For these cases, the $I-V$ characteristic is the same for all 

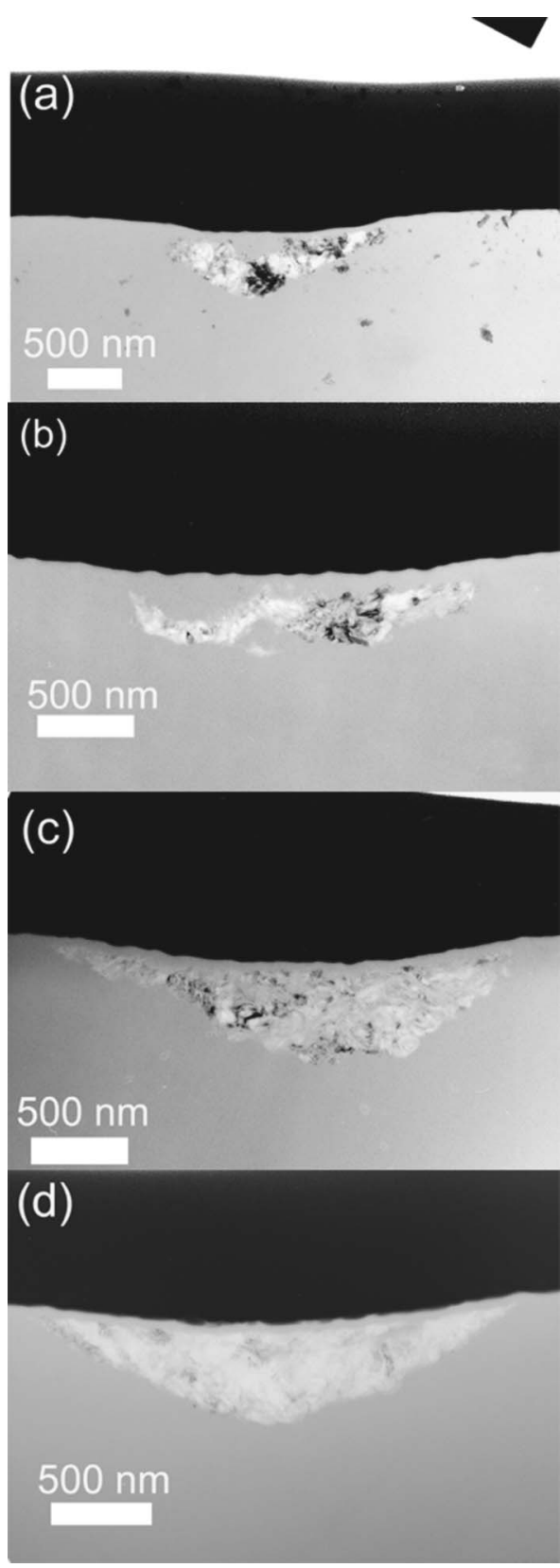

FIG. 6. Bright field XTEM images from indents in $a$-Si. The indents were created by unloading from (a) $100 \%$, (b) $90 \%$, (c) $70 \%$, and (d) $20 \%$ of the maximum load. Substantial volumes of high pressure phases are present even after unloading rapidly from the maximum load. After the pop-out event, the indents are indistinguishable and are composed solely of high pressure phases.

indents, consistent with an expected uniform microstructure and electrical properties of $a$-Si. The current is approximately two orders of magnitude lower in forward bias than in the indents that contain mostly high pressure crystalline phases commensurate with the fact that mixed high pressure phases are much more conductive than $a$-Si. These results are in agreement with the XTEM and Raman observations, which show that the phase transformed zones for these indents are completely/almost completely composed of $a$-Si.

For the indentations in $a$-Si, the $2 \mu \mathrm{m}$ thick surface $a$-Si layer forms an insulating barrier preventing measurable current flow, and hence measuring electrical characteristics is impossible. (a)

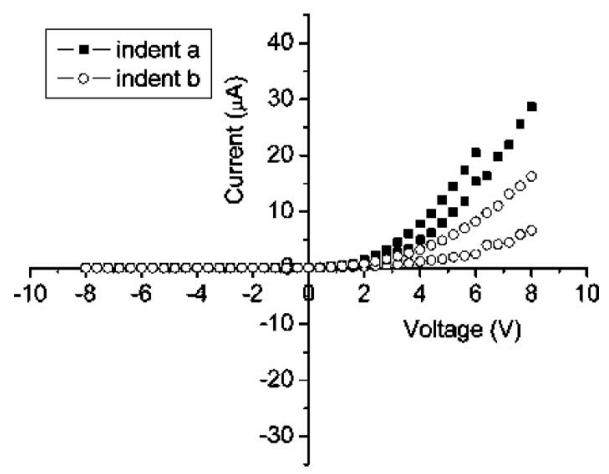

(b)

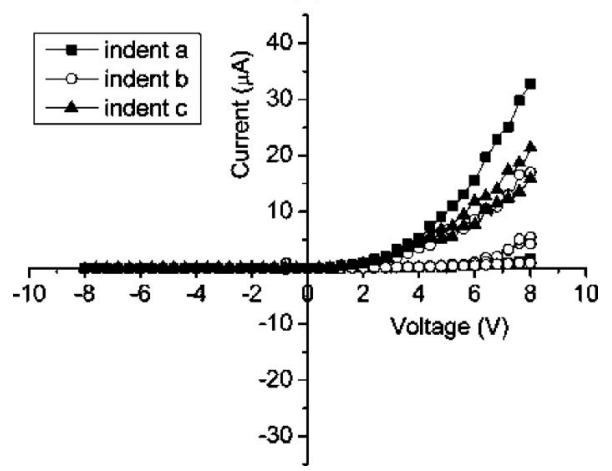

FIG. 7. Postindentation $I-V$ characteristics extracted from indents in $c$-Si (a) where slow unloading was performed and (b) for rapid unloading from $20 \%$ of the maximum load. (a) shows two measurements that were taken in different locations within the same residual indent for two different indents. (b) shows a similar set of measurements for three indents.

\section{DISCUSSION}

The data presented in the current study provide information on the transformation from $\mathrm{Si}-\mathrm{II}$ to $\mathrm{Si}$-III and $\mathrm{Si}$-XII during unloading for indentation in $a-\mathrm{Si}$ and $c$-Si and are discussed in the following three sections. The first section will discuss the evolution of these phases in the context of a nucleation and growth mechanism. The second will address possible reasons for the differences in the formation of these phases between $a$-Si and $c$-Si. Finally, the electrical measurements that were used to probe the final structures of the

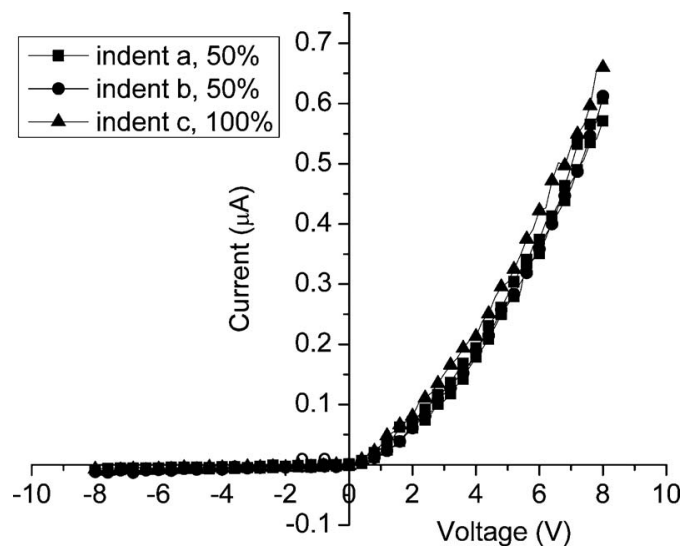

FIG. 8. Postindentation $I-V$ characteristics extracted from indents in $c$-Si where rapid unloading was performed from $50 \%$ of the maximum load and from maximum load. Only four curves are shown here for clarity, but, for all measurements made on these indents, the $I-V$ curves were similar. 
indents are discussed in more detail. As described in Sec. II, when unloading is referred to as "fast/rapid" it is at a rate of $\sim 1000 \mathrm{mN} / \mathrm{s}$ and "slow" corresponds to $\sim 0.9 \mathrm{mN} / \mathrm{s}$.

\section{A. Evolution of the high pressure phases}

It is thought that the transformation of Si-II to high pressure phases on unloading proceeds via a nucleation and growth mechanism. ${ }^{5,24}$ This infers that, in the absence of preferential nucleation sites, nucleation will occur randomly during unloading when the pressure drops below the critical value of $\sim 8 \mathrm{GPa}$. In a nucleation-limited situation, very fast pressure release may not allow high pressure phase seeds to nucleate. However, once nucleated, these seeds may grow into larger volumes of high pressure phases on subsequent unloading. Indeed, the XTEM images from the indents made in $c$-Si here, where rapid unloading occurred before the popout event, show very small volumes of Si-III/XII (undetectable by Raman), within a mostly $a$-Si phase transformed zone, and these appear to be randomly located within this volume. However, more XTEM samples would be required to confirm this proposition. For the case of indentation in $a$-Si, the high pressure phases nucleate and grow even when unloading occurs very rapidly. This observation, the fact that the high pressure phases appear to nucleate at the interface between the Si-II volume and the surrounding $a$-Si, and the substantial $a$-Si volume that is observed near the surface for fast unloading, illustrate substantial differences between high pressure phase formation in $c$-Si and $a$-Si. Further discussion of these differences follows in the next section.

Returning now to the $c$-Si case, although small seed volumes of Si-III/XII form during the early stages of unloading, they appear to grow to occupy a substantial fraction of the indent volume during a pop-out event. We propose that the formation of high pressure phases on unloading is a two step process. First, random nucleation of high pressure phases is required and these seeds appear to form randomly within $\mathrm{Si}$-II during early stages of unloading for the $c$-Si case, rather than preferentially nucleating at the $\mathrm{Si}-\mathrm{II} / a$-Si interface. Second, after seed volumes of Si-III/XII have formed, the remaining volume of Si-II becomes increasingly unstable on further pressure release and can undergo transformation to high pressure phases by rapid growth from these seeds at a critical pressure. The substantial volume of material that transforms from $\mathrm{Si}$-II to high pressure phases results in a rapid decrease in density and this causes the observed popout. If no nucleation of high pressure phase seeds has occurred, i.e., only $a$-Si, no pop-out occurs and the unstable volume of $\mathrm{Si}$-II transforms to $a$-Si. Although this process would be very difficult to observe directly, evidence does suggest that this is a plausible mechanism. First, slow unloading results in a pop-out event, which yields a phase transformed zone composed mostly of high pressure phases. This suggests that slow unloading enhances the probability of high pressure phase nucleation within Si-II during the early stages of unloading and allows the remaining volume of $\mathrm{Si}$-II to transform via growth to $\mathrm{Si}-\mathrm{III} / \mathrm{XII}$ at the critical pressure point and give rise to a pop-out. As the unloading rate increases, there is a reduced probability of nucleating high pressure phases during early stages of unloading. If no nucleation occurs at high enough unloading rates, then no rapid growth can occur at the critical pressure point and $\mathrm{Si}-\mathrm{II}$ transforms to $a$-Si during the later stages of unloading (no pop-out but an elbow in the load-unload curve). Our data support this scenario. For $c$-Si, very rapid unloading results only in the formation of $a$-Si. For slow unloading in the early stages followed by rapid unloading before a pop-out can occur, only a small number of seed volumes of high pressure phases are observed. This suggests that the unloading must be slow enough to promote random nucleation of seed volumes of high pressure phases for subsequent growth of large volumes of high pressure phases at a critical pressure (popout). Furthermore, the position of the pop-out event is relatively unaffected by the unload rate (see Fig. 2). Thus, although unload rates affect the formation of seed volumes of high pressure phases, a critical pressure also has to be reached where the remaining $\mathrm{Si}$-II transforms by rapid growth (at pop-out). Following a pop-out event, the subsequent unloading is not expected to have any effect on the phase transformed zone. In fact, following the pop-out event the entire phase transformed volume is observed by XTEM to be composed of Si-III/XII. For example, the Raman and XTEM data are indistinguishable for indents where rapid unloading occurred from $20 \%$ of the maximum load (after pop-out) or where complete unloading (slowly) occurred. This suggests that at the point of pop-out on unloading, all of the remaining $\mathrm{Si}$-II material phase transforms (to $\mathrm{Si}$-III/XII) at a critical pressure of $<8 \mathrm{GPa}$.

\section{B. Differences in high pressure phase formation in a-Si and $c$-Si}

In the case of $a$-Si, our observations indicate substantial nucleation of high pressure phases at the interface between $\mathrm{Si}-\mathrm{II}$ and the underlying $a$-Si matrix in the early stages of unloading, even for very rapid unloading. It may be that the random nature of bonding arrangements in $a$-Si can provide an increased probability of nucleation of high pressure (crystalline) phases at the interface. This could occur since the atomic spacing of Si-XII (the first phase likely to form from $\mathrm{Si}-\mathrm{II})$ is quite different for crystalline $\mathrm{Si}$-I. However, some atomic spacings in $a$-Si at the interface may better correspond to those of Si-XII and hence enhance nucleation. Thus, we suggest that the prime reason for differences in phase transformation behavior between $a$-Si and $c$-Si is the greater enhanced interface nucleation of high pressure phases in the former case. Indeed, it is clearly observed from the XTEM images taken from the indents in $c$-Si that the phases do not preferentially nucleate around this interface on unloading. Hence, in $a-\mathrm{Si}$, nucleation of high pressure seeds takes place across the entire interface area whereas in $c$-Si seeds only nucleate randomly within Si-II on unloading. This results in a much larger number of nucleation sites in $a$-Si than $c$-Si, even at very fast unloading rates.

A further observation of interest in $a$-Si is the fact that, at fast unloading rates, the near-surface appears to transform from $\mathrm{Si}-\mathrm{II}$ to $a$-Si. This suggests that the rate of growth of high pressure phases from interface seeds may not be fast 
enough: the near-surface $\mathrm{Si}$-II may then become unstable and transform to $a$-Si at the later stages of unloading. Furthermore, we note that the strain relief rate increases as the indenter tip approaches the surface during unloading (for constant unloading rate) (strain rate, $\dot{\varepsilon}=\dot{h} / h$, where $h=$ tip displacement). The difference in unloading rate between fast and slow unloading is approximately $1100(1000 \mathrm{mN} / \mathrm{s}$ for fast unloading and $0.9 \mathrm{mN} / \mathrm{s}$ for slow), which would be the corresponding difference in strain rate assuming the unloading curves are identical in shape for the two unloading rates. The increase in strain rate during unloading can be estimated from the unloading curves. For the indentations here, the strain rate increases by $\sim 3$ as the tip displacement decreases from 400 to $200 \mathrm{~nm}$. The strain rate increases further as the tip approaches the surface (the residual indents are approximately 120 to $150 \mathrm{~nm}$ deep). Thus, $a$-Si formation may be additionally favored at high strain rates. Other authors have also discussed transformation behavior in terms of strain rate rather than unloading rate. ${ }^{8,33-35}$ Indeed, Zarudi et al. ${ }^{33-35}$ suggested that differences in the stresses induced in the silicon during indentation (as a result of phase transformations) may cause differences in local strain relief rate and hence in subsequent phase transformation behavior. However, despite such subtleties, we believe that the major differences between $a$-Si and $c$-Si behavior, and also the ability of large volumes of $\mathrm{Si}-\mathrm{III} / \mathrm{XII}$ to form, are controlled by nucleation in the early stages of unloading.

\section{Electrical measurements}

Electrical measurements on indentations in silicon have been shown to be extremely sensitive to variations in the composition of the phase transformed zone. ${ }^{21,22}$ Here, postindentation measurements have been used to investigate possible differences in the indented zones of those indents made under different unloading conditions, but which appear indistinguishable by XTEM and Raman (e.g., where rapid unloading occurs at points following a pop-out event).

Following pop-out (for both $c$-Si and $a$-Si), the phase transformed zones are composed mostly of high pressure phases when examined by XTEM. Postindentation electrical measurements made on these indents do not reveal any detectable differences over and above the considerable variations within indents and between indents undergoing similar load/unload conditions (see Fig. 7). Variation at different points within an indent and between similar indents are clearly observed and this is expected as the $I-V$ characteristics are very sensitive to the phase of material in contact with the indenter tip (Si-III or $\mathrm{Si}-\mathrm{XII}$ ) and grain microstructure within the phase transformed zone. Overall, however, it is impossible to determine differences between indents unloaded rapidly from $20 \%$ (i.e., rapid unloading after pop-out) of the maximum load or unloaded slowly for the complete unloading cycle, indicating that there is no major evolution of the structure of the phase transformed zone following popout. This supports XTEM and Raman data as well as conclusions made in the previous section.

The electrical measurements made on the indentations in which rapid unloading occurred before the pop-out event
( $\geq 50 \%$ of maximum load) are identical and no variation is seen within individual indents (see Fig. 8). The measurement is insensitive to the small volumes of high pressure phases that form in the indented zone since a large proportion (>90\%) of $a$-Si is formed in these indents. The currents measured are almost two orders of magnitude smaller than those for indents with high pressure phases, reflecting the more insulating properties of $a$-Si compared with conducting/semiconducting Si-III and Si-XII phases.

\section{CONCLUSION}

By unloading rapidly at different points on the unloading portion of indentation curves in silicon, the evolution of the formation of high pressure crystalline phases can be tracked. For indentation in $c$-Si, XTEM reveals that small volumes (undetectable by Raman micro-spectroscopy) of high pressure crystalline phases form and appear to nucleate in random locations within the phase transformed zone. Only after a pop-out event occurs does the phase transformed zone contain mainly $\mathrm{Si}-\mathrm{III} / \mathrm{XII}$. We suggest a two step process for the formation of high pressure phases during unloading of $c$-Si, in which small volumes of Si-XII (and Si-III) are first randomly nucleated within Si-II during the early stages of unloading. The remaining volume of Si-II must become unstable $(<8 \mathrm{GPa})$ and have time to then transform to Si-III/ XII using the seeds as templates. The evolution of the high pressure phases in $a$-Si is more difficult to track as the nucleation and growth occur much more readily (so much so that it is difficult to form only $a-\mathrm{Si}$ ). The nucleation and growth of these phases occurs subsurface with only the near-surface containing $a$-Si if unloading occurs rapidly early on in the unload cycle.

We have shown large differences in phase transformation behavior on unloading during indentation of $a$-Si and $c$-Si. For example, rapid unloading reveals that $a$-Si transforms more readily to $\mathrm{Si}$-III/XII with $\sim 50 \%$ of the indented volume of the indentations studied here containing high pressure phases compared to the $c$-Si case where the total indented volume contained only $a$-Si. The primary reason for this seems to be that nucleation preferentially occurs at the $\mathrm{Si}-\mathrm{II} / a-\mathrm{Si}$ interface, resulting in a greater number of $\mathrm{Si}-\mathrm{XII}$ seeds compared to the relatively few randomly distributed seed volumes within the zone for the $c$-Si case. Thus, a greater volume of the phase transformed zone, for indentation in $a$-Si, is composed of these phases before a pop-out event. The variable bonding arrangements in $a$-Si contribute more favorable nucleation sites for high pressure phase formation. Furthermore, formation of Si-III/XII appears to initiate from the periphery of the metallic Si volume, but does not continue to the surface during rapid unloading, indicating that the rate of strain relief during unloading ultimately determines the volume of Si-III/XII once it has been nucleated.

Postindentation electrical measurements have been used to investigate differences in the phase transformed zones that appear to be identical by XTEM and Raman. Following popout, no major evolution of the high pressure phases is observable by this technique. Variation between indents and within the indented regions is observed, which reflects the 
random microstructure of these mixed phase volumes. In contrast, the $I-V$ characteristics measured in indents in which rapid unloading was performed before the pop-out event are identical since the volume is composed almost entirely of $a$-Si in those cases.

\section{ACKNOWLEDGMENTS}

This research was funded by the Australian Research Council and by WRiota Pty. Ltd.

${ }^{1}$ J. Z. Hu, L. D. Merkle, C. S. Menoni, and I. L. Spain, Phys. Rev. B 34, 4679 (1986).

${ }^{2}$ R. O. Piltz, J. R. Maclean, S. J. Clark, G. J. Auckland, P. D. Hatton, and J. Crain, Phys. Rev. B 52, 4072 (1995).

${ }^{3}$ J. Crain, G. J. Ackland, J. R. Maclean, R. O. Piltz, P. D. Hatton, and G. S. Pawley, Phys. Rev. B 50, 13043 (1994).

${ }^{4}$ V. Domnich, Y. Gogotsi, and S. Dub, Appl. Phys. Lett. 76, 2214 (2000).

${ }^{5}$ J. E. Bradby, J. S. Williams, J. Wong-Leung, M. V. Swain, and P. Munroe, J. Mater. Res. 16, 1500 (2001)

${ }^{6}$ J. E. Bradby, J. S. Williams, J. Wong-Leung, M. V. Swain, and P. Munroe, Appl. Phys. Lett. 77, 3749 (2000).

${ }^{7}$ D. R. Clarke, M. C. Kroll, P. D. Kirchner, R. F. Cook, and B. J. Hockey, Phys. Rev. Lett. 60, 2156 (1988).

${ }^{8}$ A. Kailer, Y. G. Gogotsi, and K. G. Nickel, J. Appl. Phys. 81, 3057 (1997).

${ }^{9}$ J. E. Bradby, J. S. Williams, and M. V. Swain, Phys. Rev. B 67, 085205 (2003).

${ }^{10}$ G. M. Pharr, W. C. Oliver, R. F. Cook, P. D. Kirchner, M. C. Kroll, T. R. Dinger, and D. R. Clarke, J. Mater. Res. 7, 961 (1992).

${ }^{11}$ G. M. Pharr, W. C. Oliver, and D. S. Harding, J. Mater. Res. 6, 1129 (1991).

${ }^{12}$ E. R. Weppelmann, J. S. Field, and M. V. Swain, J. Mater. Res. 8, 830 (1993).

${ }^{13}$ J. S. Williams, Y. Chen, J. Wong-Leung, A. Kerr, and M. V. Swain, J. Mater. Res. 14, 2338 (1999).

${ }^{14}$ Y. G. Gogotsi, V. Domnich, S. N. Dub, A. Kailer, and K. G. Nickel, J.
Appl. Phys. 15, 871 (2000).

${ }^{15}$ S. Ruffell, J. E. Bradby, and J. S. Williams, Appl. Phys. Lett. 89, 091919 (2006).

${ }^{16}$ B. Haberl, J. E. Bradby, S. Ruffell, J. S. Williams, and P. Munroe, J. Appl. Phys. 100, 013520 (2006)

${ }^{17}$ Y. Gogotsi, T. Miletich, M. Gardner, and M. Rosenberg, Rev. Sci. Instrum. 70, 4612 (1999).

${ }^{18}$ M. A. Wall and U. Dahmen, Microsc. Res. Tech. 42, 248 (1998).

${ }^{19}$ E. A. Stach et al., Microsc. Microanal. 7, 507 (2001).

${ }^{20}$ D. Ge, A. M. Minor, E. A. Stach, and J. W. Morris, Jr., Philos. Mag. 86, 4069 (2006).

${ }^{21}$ S. Ruffell, J. E. Bradby, N. Fujisawa, and J. S. Williams, J. Appl. Phys. 101, 083531 (2007).

${ }^{22}$ S. Ruffell, J. E. Bradby, and J. S. Williams, J. Mater. Res. 22, 578 (2007).

${ }^{23}$ T. Juliano, Y. Gogotsi, and V. Domnich, J. Mater. Res. 18, 1192 (2003).

${ }^{24}$ J. Jang, M. J. Lance, S. Wen, T. Y. Tsui, and G. M. Pharr, Acta Mater. 53, 1759 (2005).

${ }^{25}$ Hysitron Incorporated, “Application Note: In-situ Electrical Characterization During Nanoindentation of Crystalline Silicon" (2006).

${ }^{26}$ R. M. Langford and A. K. Petford-Long, J. Vac. Sci. Technol. A 19, 2186 (2001).

${ }^{27}$ R. M. Langford and A. K. Petford-Long, J. Vac. Sci. Technol. A 19, 982 (2001).

${ }^{28}$ T. Clarysse, P. De Wolf, H. Bender, and W. Vandervorst, J. Vac. Sci. Technol. B 14, 358 (1996).

${ }^{29}$ P. Eyben, S. Denis, T. Clarysse, and W. Vandervorst, Mater. Sci. Eng., B 102, 132 (2003).

${ }^{30}$ P. De Wolf, J. Snauwaert, L. Hellemans, T. Clarysse, W. Vandervorst, M. D'Olieslaeger, and D. Quaeyhaegens, J. Vac. Sci. Technol. A 13, 1699 (1995).

${ }^{31}$ D. M. Follstaedt, J. A. Knapp, and S. M. Myers, J. Mater. Res. 19, 338 (2004).

${ }^{32}$ V. Domnich and Y. Gogotsi, Rev. Adv. Mater. Sci. 3, 1 (2002).

${ }^{33}$ I. Zarudi, L. C. Zhang, W. C. D. Cheong, and T. X. Yu, Acta Mater. 53, 4795 (2005).

${ }^{34}$ I. Zarudi, L. C. Zhang, and M. V. Swain, J. Mater. Res. 18, 758 (2003).

${ }^{35}$ I. Zarudi, L. C. Zhang, J. Zou, and T. Vodenitcharova, J. Mater. Res. 19, 332 (2004). 